\title{
ArcheoSciences
}

Revue d'archéométrie

38 | 2014

Varia

\section{Premiers Paysans des Alpes (Lucie Martin)}

\section{Claire Delhon}

\section{OpenEdition}

\section{Journals}

\section{Édition électronique}

URL : http://journals.openedition.org/archeosciences/4310

DOI : $10.4000 /$ archeosciences. 4310

ISBN : 978-2-7535-3697-3

ISSN : 2104-3728

Éditeur

Presses universitaires de Rennes

Édition imprimée

Date de publication : 30 novembre 2014

Pagination : 251

ISBN : 978-2-7535-3689-0

ISSN : 1960-1360

\section{Référence électronique}

Claire Delhon, «Premiers Paysans des Alpes (Lucie Martin) », ArcheoSciences [En ligne], 38 | 2014, mis en ligne le 30 novembre 2014, consulté le 22 septembre 2020. URL : http://journals.openedition.org/ archeosciences/4310 ; DOI : https://doi.org/10.4000/archeosciences.4310

Article L.111-1 du Code de la propriété intellectuelle. 
Martin L., 2014. Premiers paysans des Alpes. Alimentation et agriculture au Néolithique, Rennes/Tours, Presses Universitaires de Rennes/Presses Universitaires François-Rabelais, coll. "Tables des hommes », 220 p., $20 €$.

Cet ouvrage, qui découle du travail de doctorat de son auteure, se présente comme la publication des résultats de l'analyse carpologique de quatre sites néolithiques alpins (la Grande Rivoire dans le Vercors, l'Aulp-du-Seuil en Chartreuse, le Chenet des Pierres en Tarentaise et la grotte des Balmes en Haute-Maurienne). Dans les faits, au travers de ces quatre exemples, il aborde par le biais de l'économie végétale la question fondamentale de l'occupation et de la gestion des milieux de montagne par les paysans néolithiques.

Il faut souligner la difficulté de la tâche, les problèmes de conservation des restes carpologiques en milieu sec s'ajoutant à leur faible abondance dans la plupart des sites montagnards et aux contraintes taphonomiques propres aux gisements d'altitude. L'analyse s'appuie donc sur un important et patient travail de terrain et de laboratoire, qui a permis à l'auteure de réunir un corpus suffisamment conséquent pour aborder des points cruciaux de la subsistance en milieu montagnard. En effet, les modes d'occupation, d'exploitation, de gestion des terroirs d'altitude par les agro-pasteurs néolithiques, la place de ce pan de l'économie dans un système plus complexe de gestion d'un territoire plus large ou au contraire son autonomie par rapport aux sites connus en plaine pour la même période sont des problématiques souvent débattues par les néolithiciens, alors même que des données directes sur les modalités réelles d'exploitation du milieu végétal de montagne par les néolithiques font cruellement défaut. Ce travail vient donc combler une vraie lacune pour la compréhension de cette période.

Outre la question de la cueillette de végétaux sauvages pour l'alimentation humaine, bien documentée sur tous les sites et qui démontre la large extension altitudinale des aires de récolte, la thématique de l'économie de production est largement développée. Il s'agit non seulement de la production végétale, avec une discussion fondamentale sur la possibilité d'une agriculture in situ, dans des zones souvent vues a priori (et, comme cela est bien démontré, à tort) comme impropres à la production de céréales, ou de la production animale, l'alimentation des troupeaux constituant un des moteurs majeurs de la gestion des ressources végétales.

Lucie Martin s'appuie sur l'écologie actuelle du blé engrain et de l'orge et sur des références ethnographiques pour poser l'hypothèse d'une culture de ces céréales à proximité des sites, entre 1000 et 1500 mètres d'altitude. Cette proposition semble recevable d'après les données exposées, même si l'auteure ne s'autorise pas à trancher définitivement en l'absence d'un cortège d'adventices suffisamment diversifié et abondant. Ce résultat, même s'il demande à être confirmé, va probablement changer notre perception de la place de ces gisements au sein des territoires et de l'économie Néolithique, puisqu'ils n'apparaissent plus comme forcément dépendants de sites agricoles situés en aval.

Sur le site de la Grande Rivoire, l'alimentation et les soins apportés bétail apparaissent centraux dans la constitution du corpus carpologique, qui semble ne refléter que très marginalement l'alimentation humaine. Le nourrissage du troupeau en stabulation par du fourrage d'arbre (branches feuillées et/ou fleuries) et l'apport en quantité importante de taxons inattendus comme l'if (usage sanitaire?) ou le gui (usage vétérinaire?) apportent des informations précises sur les soins aux animaux et sur les liens étroits entre la gestion des ressources végétales sauvages et la production pastorale, qui permettent eux aussi de redessiner les paysages et les territoires vécus par les Néolithiques.

Ajoutons que cette approche carpologique s'appuie sur une très bonne connaissance par l'auteure des milieux alpins d'une part et du contexte chronoculturel et socio-économique des populations étudiées d'autre part, que la lecture du texte, concis et précis, est agréable, les illustrations de bonne qualité et la bibliographie abondante, pertinente et à jour. Un catalogue carpologique des plantes domestiquées et remarquables rencontrées sur les sites étudiés, enrichi d'illustrations sera particulièrement apprécié des spécialistes. Enfin un glossaire permettra à tous les lecteurs, grand public et étudiants compris, d'aborder cet ouvrage sans peine.

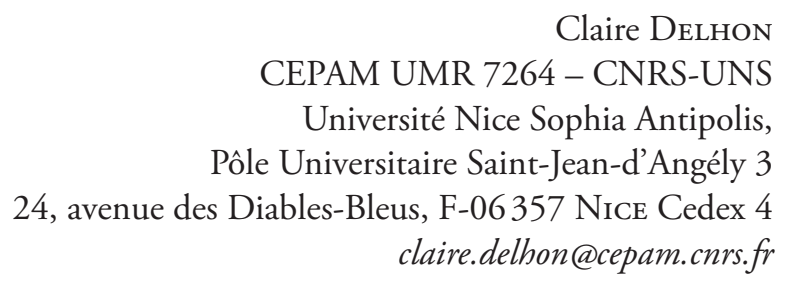
claire.delhon@cepam.cnrs.fr 